\title{
Applications, Challenges and Protocols of MANETs: A Review
}

\author{
K. Sri Varsha1), S. Naga Mallik Raj2)
}

\begin{abstract}
Portable means moving and specially ad hoc means brief with no settled framework so versatile impromptu systems are a sort of impermanent systems in which hubs are moving with no settled foundation or unified management[1-3]. Mobile Ad-hoc arranges have been broadly looked into for a long time. Impromptu system is a gathering of hubs that is associated through a remote medium shaping quickly evolving topologies. Remote gadgets are always developing in correspondence field having all the more figuring speed and various highlights, while contracting in weight and size. Versatile specially appointed systems (MANETs) speak to complex conveyed frameworks that involve remote portable hubs that can openly and powerfully self-arrange into self-assertive and transitory system topologies. Individuals and gadgets are permitted to flawlessly internetwork in regions with no prior correspondence framework, e.g., fiasco recuperation conditions. Steering in Mobile Ad-hoc Networks is a testing undertaking because of its incessant alteration in topologies. We examine in this paper directing convention, difficulties and security of specially appointed systems.
\end{abstract}

Keywords : Characteristics, Routing Protocols, MANETs, Challenges, Protection.

\section{Introduction}

The multiplication of portable figuring and specialized gadgets (e.g., phones, tablets, handheld advanced gadgets, individual computerized partners) is driving a progressive change in our data society[4]. We are moving from the customary wired interchanges to remote correspondences. Remote systems comprise of various hubs which speak with each other over a remote channel. There are right now two varieties of portable remote systems: foundation and framework less systems. The foundation systems, in which cell phones speak with base stations that are associated with settled system framework. Every hub in the foundation systems is inside the scope of a settled access point like base station. Utilizations of this sort incorporate cell phone and remote neighborhood. The other kind of remote system, foundation less systems knows as MANETs[2]. These systems have no settled

Received(November 10, 2017), Review Result(1st: December 1, 2017, 2nd: December 31, 2017), Accepted(January 5, 2018)

1) (Professor) Department of Computer Science and Engineering, Vignan's Institute of Information Technology, Visakhapatnam, AP.

email: varsha.kongara@gmail.com

2) (Professor, Corresponding Author) Department of Computer Science and Engineering, Vignan's Institute of Information Technology, Visakhapatnam, AP.

email: mallikblue@gmail.com 
access focuses while each hub could be host or switch. All hubs are equipped for development and can be associated progressively in self-assertive way. These systems are self configurable[4] and self-sufficient frameworks comprising of switches and has. These hubs are compelled in control utilization, transmission capacity, and calculation control[2].

MANETs need focal organization and earlier association, so the security issues are extraordinary and hence requires diverse security components than in customary systems. Remote connections in MANETs make them more inclined to assaults. It is less demanding for programmers to assault these systems effectively and in this manner access classified data. They can likewise specifically assault the system to erase messages, include malignant messages, or take on the appearance of a hub. This damages the system objectives of accessibility, respectability, secrecy, genuineness and approval[5]. MANET require a to a great degree adaptable innovation for building up correspondences in circumstances which request a completely decentralized system with no settled base stations, for example, front lines amid wars, military applications, and other crisis inquiry and safeguard circumstances at the season of catastrophes. Directing in impromptu systems faces extra issues and difficulties when contrasted with steering in customary wired systems. In this paper, we examine distinctive directing conventions and difficulties of MANETs.

This expansion is reflected by advancement in the numbers and sorts of customers of frameworks from examiners and associations to families and individuals in general use. Since their ascent in the 1970s, remote frameworks have ended up being continuously outstanding in the enrolling business. This is particularly legitimate inside the earlier decade which has seen remote frameworks being changed in accordance with enable movability. There are starting at now two assortments of convenient remote frameworks. The first is known as infrastructured frameworks, i.e., those frameworks with settled and wired gateways. The augmentations for these frameworks are known as base stations. A flexible unit inside these frameworks interfaces with, and talks with, the nearest base station that is inside its correspondence traverse. Average uses of this sort of framework consolidate once remote neighborhood (WLANs). The second kind of convenient remote framework is the foundation less flexible framework, usually known as an off the cuff framework. Foundation less frameworks have no settled switches; all centers are prepared for improvement and can be related capably subjectively. Centers of these frameworks fill in as switches which find and keep up courses to various centers in the framework.

Off the cuff frameworks outline all of a sudden without a need of an establishment or concentrated controller. This sort of dispersed structure interprets that each center point, or customer, in the framework can go about as a data endpoint or widely appealing repeater. Along these lines, all customers participate to upgrade the faithful nature of framework exchanges. These sorts of 
frameworks are in like manner commonly alluded to as "work frameworks" in light of the way that the topology of framework exchanges takes after a work. The redundant correspondence courses gave by exceptionally selected work organizes certainly improve adjustment to non-basic disappointment for the framework. Moreover, the limit with respect to data bundles to "bob" beginning with one customer then onto the following enough grows the framework scope area and gives a response for overcome non-visible pathway (LOS) issues. Flexible applications show additional troubles for work organizes as changes to the framework topology are speedy and in all cases. Such circumstances require the use of Mobile Ad hoc Networking (MANET) development to ensure correspondence courses are revived quickly and decisively. MANETs are self-surrounding, self-kept up, and self-retouching, thinking about unprecedented framework flexibility. While MANETs can be absolutely autonomous, they can moreover be settling to an IP-based worldwide or neighborhood orchestrates (e.g. Web or private frameworks). These are implied as Hybrid MANETs.

\section{Characteristics and Applications}

\subsection{Accompanying attributes of the MANETs}

1. Independent conduct in MANETs: Every hub goes about as both host and router[5]. It implies that a hub has capacity of host and can likewise perform exchanging capacities as switch so endpoints and switches are undefined.

2. Multi-jump Broadcast: When a source hub and goal hub for a communication is away from the broadcast expand, the MANETs are fit for multi-bounce transmission. While conveying information bundles from a source to its goal out of the immediate remote transmission extend, the parcels must be sent through at least one middle of the road hubs.

3. Disseminated Nature of Procedure: As a brought together control is truant here, the control and operation of the system is circulated among the hubs. The hubs should team up to actualize numerous works for the most part security and steering.

4. Energetically Evolving Topology: Due to portable hubs, the adjustment in topology is continuous and dynamic in nature[7]. The availability among the hubs may fluctuate with time and powerfully set up steering among them as they move about.

5. Inferior Connection Limit: The dependability, adaptability, proficiency and limit of remote connections are regularly substandard when contrasted and wired connections. One end to end way can be shared by a few sessions. The terminals convey through which channel is liable to clamor, blurring, obstruction and has less transmission capacity than a wired system. This demonstrates the 
fluctuating connection data transmission of remote connections.

6. Symmetric Condition: All hubs have indistinguishable highlights with comparable obligations and abilities. Each hub can work as a switch or have and henceforth it frames totally symmetric condition.

7. Nonexistence of Infrastructure: Ad-hoc organizes should work freely of any settled framework.

\subsection{Distinctive Applications Comprises}

1. Military War Zone: Military gear at the present regularly have a type of PC equipment[6][7]. Through impromptu systems administration, the military could take the upside of typical system innovation to keep up a data organize among the vehicles, officers and military head quarters. Essentially the procedures of specially appointed systems originated from this field.

2. Commercial Part: Ad hoc can be used as a piece of emergency/spare operations for trademark upheavals mitigation tries, e.g. in fire, surge, or tremor. Spare operations must happen where non-existing or hurt trades system and quick course of action of a correspondence compose is required. Information is passed on beginning with one spare associate then onto the following.

3. Local level: Ad hoc frameworks can self-rulingly associate a minute and brief sight and sound framework using scratch pad PCs or palmtop PCs to spread and offer information among individuals at a meeting. Another legitimate neighborhood level application might be in home frameworks where contraptions can pass on particularly to exchange information.

4. Personal Area Network (PAN): Short-go MANET can streamline the connection between networks with various mobile phones (for instance, a wireless, convenient PCs, and wearable computers)[7]. Standard wired connections are supplanted with remote affiliations.

\section{Routing Protocols}

Directing conventions characterize an arrangement of principles which oversees the trip of message parcels from source to goal in a system[4]. In MANET, there are diverse sorts of directing conventions each of them is connected by the system conditions. Figure 1 demonstrates the fundamental characterization of the steering conventions in MANETs.

\subsection{Proactive Routing Protocols}

Proactive steering conventions are additionally called as table driven directing conventions. In this 
every hub keeps up directing table which contains data about the system topology even without requiring it. The steering tables are refreshed occasionally at whatever points the system topology changes[2]. Proactive conventions are not suitable for extensive systems as they have to keep up hub passages for every single hub in the directing table of each hub. There are different proactive steering conventions. Case: DSDV, OLSR, WRP and so forth.

\subsection{Receptive Routing Protocols}

Receptive directing convention is otherwise called on request steering convention. In this kind of convention, course is found at whatever point it is required. Hubs start course disclosure when requested. A course is procured by the start of a course disclosure process by the source hub. These steering conventions have two noteworthy segments:

1. Route disclosure: In this stage source hub starts course revelation on request premise. Source hubs counsels its course reserve for the accessible course from source to goal generally if the course is absent it starts course disclosure. The parcel of the source hub incorporates the address of the goal hub too address of the moderate hubs to the goal.

2. Route support: Owing to active topology of the scheme instances of the course disappointment between the hubs emerges because of connection breakage and so on, so course upkeep is required. Responsive conventions have affirmation system because of which course upkeep is conceivable. There are different receptive steering conventions.

\subsection{Half and half Routing Protocol:}

This sort of convention is an exchange off among practical and open customs. Proactive customs have all the more overhead and less lethargy while responsive conventions have not so much overhead but rather more latency[4]. Subsequently a Hybrid custom is depended upon to beat the inadequacies of responsive coordinating conventions. This custom is a mix of both proactive and open coordinating convention. It utilizes the on request course of action of responsive custom and the table assist fragment of proactive convention with a particular true objective with avoiding dormancy and overhead issues in the structure. Cross breed convention is fitting for wide structures where liberal measures of focus focuses are available. In this, broad structure is confined into a game-plan of zones where controlling inside the zone is finished by using practical advance and exterior zone organizing is finished using receptive method. There are differing cream planning customs for MANET like ZRP, SHRP and so on. 


\subsection{Difficulties}

Despite numerous qualities, the MANET presents a few difficulties that must be examined deliberately. These are following:

1. Steering: Due to the continually changing topology in specially appointed systems, directing the parcels between any combine of hubs turns into a testing errand. The vast majority of the conventions in view of receptive directing rather than proactive steering. Multi cast directing is another test as the multi cast tree isn't [7]. Courses between the hubs may have numerous bounces, which is more mind boggling than the single jump correspondence.

2. Safety and Dependability: Impromptus systems have security issues e.g. awful neighbor handing-off packets[3]. The element of appropriated procedure needs varied strategy of confirmation. Further, remote connection includes likewise present dependability issues, in light of the restricted transmission run, the communicate idea of the remote medium (e.g. concealed terminal issue), portability instigated bundle trouble, and in order communication mistakes.

3. Between systems administration: Notwithstanding the correspondence inside a specially appointed system, between systems administration amongst MANET and framework systems (for the most part IP based) is regularly expected much of the time. The steering conventions conjunction in such a cell phone is a test for versatility organization[3].

4. Power Consumption: The vast majority of the trivial cell phones, the correspondence connected capacities ought to be streamlined for lean authority utilization. Power preservation and power-mindful steering have to be measured.

\section{Security in MANETs.}

We examine security criteria and assaults which are following:

1. Accessibility-The term Accessibility suggests that a center point should keep up its ability to give all the created organizations paying little regard to its security state. This security worldview is harmed on a very basic level in the midst of the foreswearing of-advantage strikes, in which centers in the framework can be the attack target and in like manner some immature center points make a segment of the framework organizations out of reach.

2. Confidentiality-infers that fastidious data is available to the people who contain is endorsed to get to it. By the day's end, in order to keep up the arrangement of some private information, we have to stay furtive from all matters that were not affirmed to get to them[4].

3. Verification Ensures that individuals in correspondence are true blue. It is basic for the 
correspondence components to exhibit their lifestyles stated by means of couple of methods. If there isn't such an affirmation instrument, the enemy could go about as a positive center and subsequently get to mystery information, or even install a number of fraud letters to concern the usual framework tasks.

4. Non-foreswearing guarantees that the dispatcher and the gatherer of a communication can't reject that they never send or got a note[1]. This is significant when we need to isolate if a center point with some strange lead is exchanged off or not: if a center point sees that it has gotten a mixed up message, it would then have the capacity to use that message as a proof to tell diverse centers that the center point passing on the shocking meaning would be haggled.

5. Authorization: No one else can pronounce to be another endorsed part to take in any significant information. It is all things considered used to dole out unlike contact to dissimilar stage of customers.

6. Attacks using production of phony coordinating messages is named as creation[6]. Such sorts of ambushes are hard to perceive.

\section{Attacks on MANETs:}

There are different sorts of assaults on specially appointed system which are following:

1. Location Disclosure: Location divulgence is an assault that objectives the security prerequisites of an impromptu network[5]. By utilizing movement investigation strategies, more straightforward examining and checking approaches, an aggressor can identify the area of a hub, or the structure of the entirety organize.

2. Black Hole: In a dark gap assault a vindictive hub infuses false course answers to the course asks, declaring it as having the most limited way to a destination[3]. These phony answers can be manufactured to occupy arrange movement through the malignant hub for essentially to pull in all activity towards it in request to play out a disavowal of administration assault by disposing of the got parcels.

3. Wormhole: The current assault is a standout amongst the most effective displayed here since it includes the collaboration between two malignant hubs that take an interest in the system. One assailant, e.g. hub A, catches steering activity at one purpose of the system and passages them to another point in the arrange, to hub B, for instance, that offers a private correspondence interface[6]. Hub B at that point specifically infuses burrowed movement once again into the system. The association between the hubs that have set up courses over the wormhole connect is totally controlled by the two contriving assailants. The parcel rope is the answer for this assault. 
4. Blackmail: This assault is significant against steering conventions those utilization systems for the recognizable proof of noxious hubs and proliferate messages that endeavor to boycott the assailant. An aggressor may build such detailing messages and endeavor to segregate authentic hubs from the organize. The non-revocation security criteria can end up being helpful as it ties a hub to the messages it produced.

5. Denial of Service: Contradiction of administration assaults go for the total interruption of the steering capacity furthermore, in this manner the whole operation of the specially appointed system. In a steering table flood assault the vindictive hub surges the system with sham course creation parcels keeping in mind the end goal to devour the assets of the taking an interest hubs and upset the foundation of legitimate courses.

6. Routing Table Poisoning: Routing conventions keep up tables that hold data with respect to courses of the system. In this sort of assaults, the vindictive hubs produce and send created flagging activity, or adjust true blue messages from different hubs, so as to include false sections in the tables of the taking part hubs. For instance, an aggressor can send directing updates that don't compare to genuine modifications in the topology of the specially appointed system. Directing table harming assaults can have non-ideal courses, directing circles, bottlenecks, and notwithstanding assigning certain parts of the network[6].

\section{Difficulties of MANET}

When scope of uses for portable specially appointed system is increment, their utilizations are additionally expanded however there are a few downsides of utilizing the current network innovation. There are still a few difficulties and issues that ought to be worry in future research. Given underneath are some intricacy and difficulties that are look by utilizing the current networks. The adaptability of current network increment as it is utilized as a part of secure systems so every hub ready to deal with the general system and satisfy their obligation. In this situation sender gadget is not remain the end framework. It ought to be going about as a switch or middle of the road gadget. There is no brought together system for information conveyance. Each versatile sends information and furthermore go about as a switch to spread message. Each hub sent refresh to different hubs, this will build the system movement overhead and that is the reason there might be chance for circle framing by altering the topology. Every hub goes about as independent framework in arrange subsequently it is gear for RF to get these abilities that Forms lopsided connection. It utilizes no switch between these hubs for conveyance of parcel. Each hub goes about as a switch in current networks[2][7]. Nature of Service in current network is exceptionally intricate issue which was a 
noteworthy worry for prospect specialists.

\section{Conclusion}

In this paper, a review of dissimilar safety concerns, assaults on corporal, information and framework layers and moreover give concern plans. Distinctive guiding traditions analyzed in the paper are astoundingly supportive and effectual for fresh researchers to perceive energy issues for move ask about. Various new coordinating traditions are proposed nowadays yet in the meantime present is an untie investigate concern what tradition demonstrates best direct in which circumstances. A lot of responsibility has been made in this ground however a couple of open issues and issues ought to be tended to.

\section{References}

[1] Abdalla, G.M.T., Abu-Rgheff, M.A. and Senouci, S.M., Current trends in vehicular ad-hoc networks, IEEE Global Information Infrastructure Symposium, (2007), July 2-6; Marrakech, Morocco.

[2] Abolhasan, M., Wysocki, T. and Dutkiewicz, E., A Review of Routing Protocols for Mobile Ad Hoc Networks, Ad Hoc Networks, (2004), Vol.2, pp.1-22.

[3] Al-Omari, S.A.K. and Sumari, P., An Overview of Mobile Ad Hoc Networks for the Existing Protocols and Applications, (2010), arXiv Preprint, arXiv:1003.3565

[4] Biradar, R.C. and Manvi, S.S., Agent-Driven Backbone Ring-Based Reliable Multicast Routing in Mobile Ad Hoc Networks. Communications IET, (2011), Vol.5, pp.172-189.

[5] Chlamtac, I., Conti, M. and Liu, J.J.-N., Mobile ad Hoc Networking: Imperatives and Challenges, Ad Hoc Networks, (2003), Vol.1, pp.13-64, http://dx.doi.org/10.1016/S1570-8705(03)00013-1

[6] De Morais Cordeiro, C. and Agrawal, D.P., Mobile Ad Hoc Networking, Center for Distributed and Mobile Computing, ECECS, University of Cincinnati, USA, (2002)

[7] Gagandeep, A. and Kumar, P., Analysis of Different Security Attacks in MANETs on Protocol Stack A-Review, International Journal of Engineering and Advanced Technology (IJEAT), (2012), Vol.1, pp. $269-275$. 